\title{
Perfil epidemiológico de zoonoses nos municípios afetados diretamente pela Usina Hidrelétrica Estreito
}

\section{Epidemiological profile of zoonoses in cities directly affected by the Mill Dam Estreito}

Helierson Gomes - Mestre em Ciências Ambientais e Saúde, pela PUC-GO, 2014. Professor efetivo da Universidade do Estado do Pará/UEPA, Belém-PA. E-mail: profhelierson@ gmail.com

Andrielly Gomes de Jesus - Mestranda em Saúde da Família, pela Universidade Estácio de Sá, Professora substituta da Universidade do Estado do Pará/UEPA, Belém-PA. E-mail: andriellygm@gmail.com

Nelson Jorge da Silva Jr. - Doutor em Zoologia, pela Brigham Young University (USA), 1995. Professor da Pontifícia Universidade Católica de Goiás, Goiânia-GO. E-mail: nelson.jorge.silvajr@gmail.com.

\begin{abstract}
Resumo
Diante das constantes ações antrópicas, resultando em desequilíbrios nos mais diversos segmentos, como ambiental, social e de saúde pública, realizouse um estudo com o objetivo de avaliar o impacto da construção da UHE Estreito, no estado do Maranhão, Brasil, na incidência de zoonoses nos municípios de Carolina e Estreito (MA). Adotouse uma metodologia para obtenção das médias de incidência no período que compreende a fase préimplantação da usina (2001-2005), a fase durante a implantação (2006-2010) e a fase pós-implantação (2011-2012), enfatizando as zoonoses de importância epidemiológica na região, como é o caso da Dengue, Leishmaniose Tegumentar e Visceral, Doença de Chagas, Febre Amarela e Malária. Como resultado, a Dengue, com 907 casos, foi a morbidade com maior prevalência, sendo a fase durante a construção da UHE a de maior incidência, com 1,75\% na cidade de Carolina e 1,59\% em Estreito, seguida da Leishmaniose Tegumentar americana, com 175 casos notificados, também apresentando maior incidência no período durante as obras. A doença de chagas, febre amarela e malária não apresentaram casos notificados na região de estudo. Em conclusão, os dados obtidos neste estudo não sugerem a interferência direta entre a construção da UHE e alterações epidemiológicas consideráveis das morbidades estudadas, sugerindo a realização de mais estudos após o período de enchimento do lago.
\end{abstract}

\section{Palavras-chave}

Hidrelétricas. Saúde Pública. Epidemiologia. Zoonoses.

\begin{abstract}
By constant anthropic actions, resulting in several segments imbalance, such as, environmental, social and health service, we carried out a study to evaluate the influence that the construction of HPP Estreito, in Maranhão, Brazil, had on the rate of zoonotic diseases in the provinces of Carolina and Estreito (MA). As a method, we compared the records of the average incidence, in each province, covering the period that preceded the power plant's construction (2001-2005), the period throughout the construction (2006-2010) and after the building was completed (2011-2012); highlighting epidemiological zoonotic diseases, such as Dengue, leishmaniasis, visceral leishmaniasis, Chagas disease, yellow fever and malaria. Dengue, with 907 incidents had the highest morbidity rate. In the course of the HPP construction, the province of Carolina presented an increase of $1.75 \%$ in its dengue cases and Estreito presented an increase of $1.59 \%$. Leishmaniasis, with 175 cases notified, presented a higher occurrence in the period during the construction. Chagas disease, yellow fever and malaria had not presented any cases, in the provinces where the study was held. The research does not suggest that there is a direct connection between the HPP construction and epidemiological changes considered in this research.
\end{abstract}

\section{Keywords}

Hydroelectric. Health Service. Epidemiology. Zoonosis. 


\section{INTRODUÇÃO}

É indiscutível que nos dias atuais a energia elétrica seja indispensável na vida das pessoas. Em um país em que a matriz energética é eminentemente hidráulica, não é novidade que a cada dia surja o lançamento de novos projetos para construção de usinas hidrelétricas (UHE). No mundo, são mais de 45.000 grandes usinas, e só no Brasil são mais de 500 hidrelétricas, com estimativa, segundo a Eletrobras de lançamento de aproximadamente 494 projetos de novos empreendimentos até 2015. Apesar de 90\% de toda energia utilizada ser de origem hidráulica, esse quantitativo é oriundo de apenas $42 \%$ de todo o potencial hidrelétrico existente no país (ANEEL, 2002; AMARAL et al., 2010).

Por volta de outubro de 2012, entrou em operação uma das maiores obras do Programa de Aceleração do Crescimento (PAC I), do Governo Federal, que é a Usina Hidrelétrica (UHE) Estreito, instalada no rio Tocantins, entre os municípios de Estreito (MA) e Aguiarnópolis (TO), com capacidade instalada de 1.087 MW. Seu reservatório possui uma área inundada de $400 \mathrm{~km}^{2}$ e extensão de $260 \mathrm{~km}^{2}$, abrangendo diretamente um total de 12 municípios: Carolina e Estreito, no estado do Maranhão; Aguiarnópolis, Babaçulândia, Barra do Ouro, Darcinópolis, Filadélfia, Goiatins, Itapiratins, Palmeirante, Palmeiras do Tocantins e Tupiratins, no estado do Tocantins (CESTE, 2013).

Diversas são as consequências provocadas pela construção desses empreendimentos, consequências estas que se dividem a cada etapa da implantação dessas usinas. Os abalos sociais e urbanos, principalmente no período de construção, quando o deslocamento populacional é intenso para essas regiões, gerando diversos transtornos, como o aumento da inflação local, tráfico de drogas, violência, prostituição, acidentes, crescimento desordenado de áreas periféricas, entre outros (FERRETE et al., 2004; OLIVEIRA et al., 2006).

Após o término das obras, e com o enchimento total do lago reservatório, destacam-se outros fatores, como a alteração na ocorrência de algumas doenças e a notória e devastadora destruição da biodiversidade de toda a área afetada pelo lago reservatório da usina, modificando todo o ecossistema local, gerando alterações macro e microclimáticas, provocando, assim, um período de instabilidade ambiental que pode levar décadas para retomar o equilíbrio natural (OLIVEIRA et al., 2006; DACHERY et al., 2010). 


\section{HIDRELÉTRICAS E SAÚDE PÚBLICA}

Fatores como o não desmatamento prévio da vegetação às margens da área a ser alagada e a morte de diversos animais, provocada pelo alagamento, resultam em um acúmulo de matéria orgânica no interior do lago, gerando uma produção excessiva de sulfito de hidrogênio, fazendo com que ocorra a morte de mais animais, entre eles os de vida aquática por hipóxia, alterando ainda mais a qualidade dessa água, favorecendo o aumento nos índices de casos de doenças relacionadas ao consumo dessa água (OLIVEIRA et al., 2006).

Além dos problemas de saúde decorrentes da mobilização humana desordenada, DSTs, violência, alcoolismo, entre outros fatores, ocorre uma grande mobilidade faunística provocada pelo avanço da água e alagamento dos habitats naturais. Esses animais são obrigados a migrar em busca de novos habitats e alimentos, levando-os a se deslocar em direção às cidades próximas, devido à grande oferta de alimentos decorrente dos depósitos de lixo, aumentando a possibilidade de acidentes causados por picadas de animais peçonhentos e o surgimento de diversas doenças, por esses animais serem potenciais portadores de diversas zoonoses (SILVA JR et al., 2005).

A mudança brusca no ecossistema local pode gerar o surgimento de diversas doenças antes não endêmicas na região. Fatores como o alagamento de matéria orgânica, mudança microclimática e mobilização humana e faunística nessas regiões são fatores essenciais para a disseminação de doenças, em especial, as zoonóticas transmitidas por vetores, haja vista que tais condições são ideais para a proliferação de artrópodes. Conforme observado em pesquisas realizadas em regiões impactadas por hidrelétricas, algumas espécies como o mosquito do gênero Mansonia chegou a uma densidade de 600 picadas/pessoa/hora, fato associado ao movimento humano e faunístico, criando condições ideais para a disseminação de doenças (COUTO, 1996; OLIVEIRA et al., 2006; GOMES, H., 2014).

Os critérios utilizados pelos órgãos ambientais - estes sempre ligados ao governo - para a liberação desses empreendimentos, assim como a pouca efetividade das políticas públicas em prol de estudos epidemiológicos nessas regiões, serão sempre temas de sérias discussões, pelo fato de que essas UHEs abalam drasticamente todo o ecossistema local, mas, por outro lado, até o momento são a forma menos destrutiva e com capacidade de suprir as demandas no que se refere à energia elétrica, levando a um questionamento sobre a importância de pesquisas, no intuito de descobrir novas fontes de energia capazes de suprir as demandas e gerar menos impactos (OLIVEIRA, et al., 2006; ANEEL, 2002). 
Com objetivo de avaliar a influência da construção da UHE Estreito na incidência de zoonoses nos municípios de Carolina e Estreito, no estado do Maranhão, foi realizado um levantamento do perfil epidemiológico das zoonoses de importância epidemiológica na região, como é o caso da Dengue, Leishmaniose Tegumentar e Visceral, Febre Amarela, Doença de Chagas e Malária.

\section{MATERIAIS E MÉTODOS}

A área de estudo engloba os municípios de Carolina e Estreito, ambos no estado do Maranhão, diretamente impactados pela implantação da UHE Estreito - Estreito por ser cidade sede do canteiro de obras e Carolina por ser a maior cidade a montante, e com maior área territorial a ser alagada.

Para obtenção dos dados, foi realizada uma pesquisa documental, tendo como fontes o Instituto Brasileiro de Geografia Estatística (IBGE) para coleta de informações referentes a estatísticas e dados geográficos, o Sistema de Informação de Agravos Notificados (SINAN) e o DATASUS.

Os dados foram coletados em três períodos. O primeiro período é referente à época que antecede a construção da usina (2001-2005), o segundo compreende a fase de construção e implantação (2006-2010), e o terceiro período é referente à fase após a implantação da usina hidrelétrica, já com toda a área do lago sob inundação (2011-2012). Para haver uma padronização geral, foram coletados os casos confirmados (CC) e a incidência (I).

A comparação das médias de ocorrência de cada zoonose foi realizada utilizando o teste de Análise de Variância (ANOVA), considerando tanto os valores absolutos como também os valores de incidência. Posteriormente, foi realizado o teste de Tukey, considerando o intervalo de confiança igual a 0,95. A normalidade dos dados e homogeneidade de variância dos dados foi testada através dos testes de Kolmogorov-Smirnov e Levene, respectivamente (ZAR, 1999). Os valores aceitáveis para $p$, quando as diferenças entre as médias estatisticamente significativas foram de $p \geq 0,01$ e $p \leq 0,05$ (ZAR, 1999).

\section{RESULTADOS/DISCUSSÃO}

Com referência à incidência de zoonoses e a possível associação com a implantação da UHE Estreito, nos municípios de Carolina e Estreito (MA), apresentam-se dados referentes às fases antes das obras, durante as obras e pósalagamento da usina, no período 2001 a 2012, respectivamente (Tabela 1).

Novos Cadernos NAEA • v. 17 n. $2 \cdot$ p. 287-301 • dez. 2014 


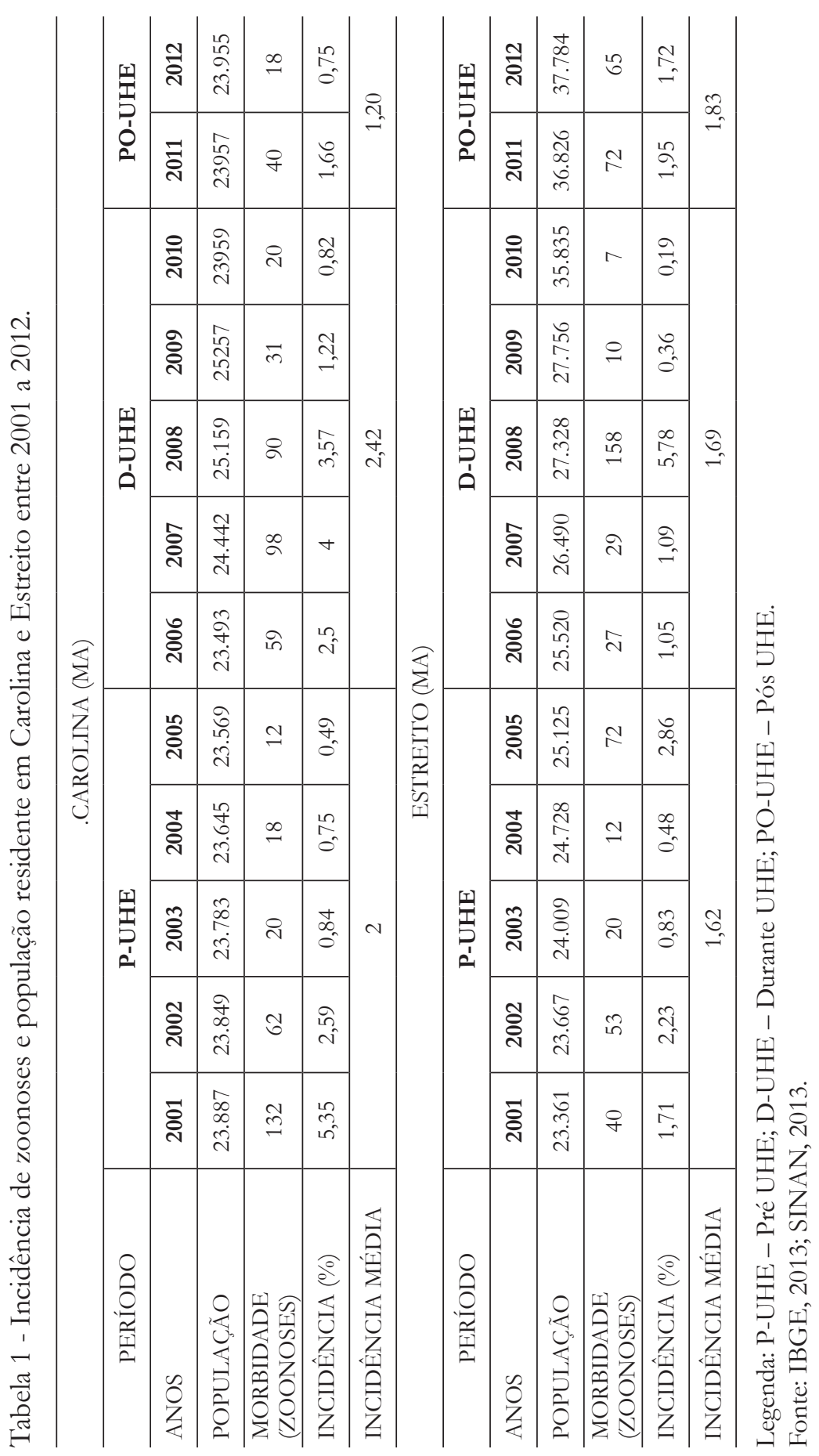

Novos Cadernos NAEA • v. 17 n. 2 • p. 287-301 • dez. 2014 
Nos municípios estudados houve um aumento populacional de 36,9\% (17.335 habitantes), com a grande maioria concentrada na cidade de Estreito (MA) 89,8\% (14.423), cidade sede das obras civis, contra 1.764 pessoas em Carolina $(10,17 \%)$, maior cidade a montante, e com maior área territorial submersa pelo lago reservatório. A cidade de Estreito, mesmo apresentando variação considerável de habitantes em relação à Carolina $(p=0,022 / p<0,05)$, não apresentou valores com as dimensões da UHE Tucuruí, nem com a mais recente, UHE Belo Monte, que em dois anos a região já conta com um aumento populacional que gira entorno dos 45 mil novos habitantes (PEDUZZI, 2013). Entretanto, não foi possível obter dados referentes à procedência, instalação e tempo de permanência dos trabalhadores nas cidades de lotação, junto ao consórcio responsável pela obra.

Carolina, por sua vez, após o término das obras sofreu nova regressão populacional devido a grande parte das pessoas terem partido junto com as empresas às quais prestavam serviços. Estreito, no entanto, por possuir melhor infraestrutura e maior poder econômico advindo da UHE, e pelo intenso potencial financeiro gerado pela malha rodoviária, permaneceu sem uma queda brusca na população. A seguir, apresenta-se a análise dos dados sobre a incidência de zoonoses na área de estudo (Figuras 1 e 2).

Figura 1 - Incidência de zoonoses no município de Carolina de 2001 a 2012.

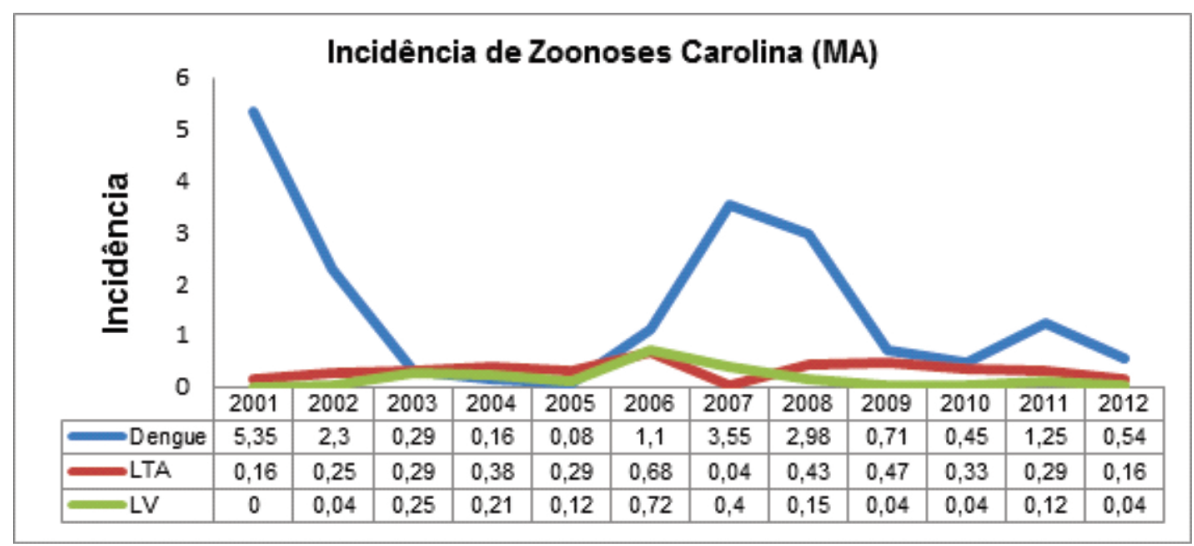

Fonte: SINAN, 2013. 
Figura 2 - Incidência de zoonoses no município de Estreito, de 2001 a 2012.

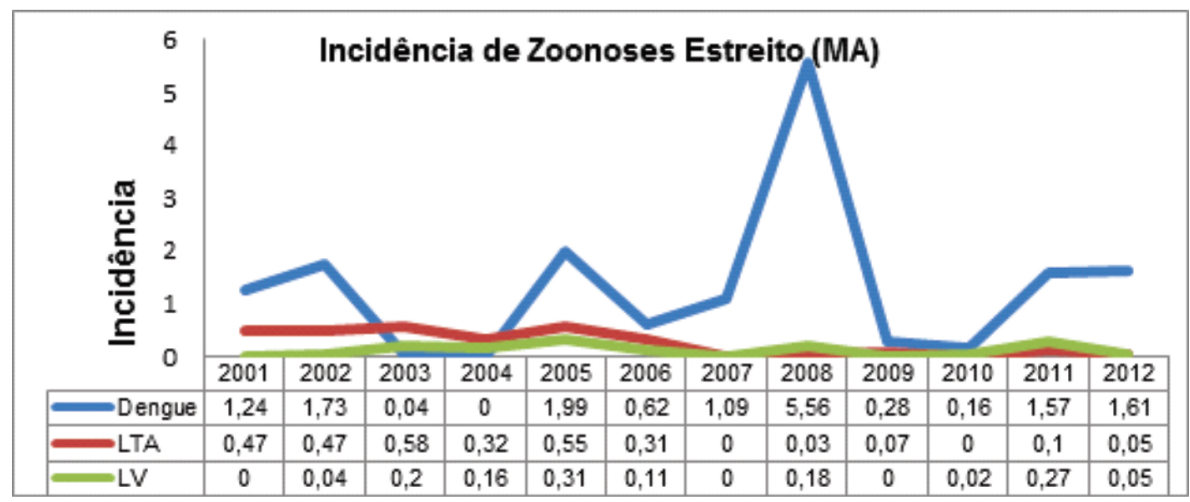

Fonte: SINAN, 2013.

\section{Dengue}

Em grande parte dos estudos epidemiológicos em regiões impactadas por hidrelétricas, a Dengue apresentou o maior número de casos notificados, como em Corumbá IV, onde foram notificados mais de 2 mil casos e, mais recentemente, em Cana Brava (2012), onde todas as cidades estudadas apresentaram casos da doença. Estreito e Carolina também tiveram a doença como uma das mais incidentes, com destaque para os anos de 2001, 2007 e 2008, com acentuada elevação dos casos; segundo Brasil (2010), graças à inserção das novas formas virais - o tipo 3 (2001) e o tipo 4 (2007/2008) - na cadeia epidemiológica da doença, adicionadas aos casos das formas virais já existentes, contribui para uma epidemia nesses períodos, porém em âmbito nacional, não ocorrendo apenas na região estudada, e não podendo se associar especificamente as obras da UHE como fator responsável por este fato.

Os dois municípios encontram-se na situação de regiões de alerta, no que se refere ao índice de infestação predial (IIP), que indica o percentual de imóveis com presença de larvas do vetor da Dengue em relação ao total de imóveis pesquisados (BRASIL, 2005). Ambas as cidades apresentam uma situação entre 1 e 3,9\% de IIP, índices acima dos valores ideais, que é de até 1\% (SINAN, 2013) (Figura 3). 
Figura 3 - Taxa de incidência de Dengue.

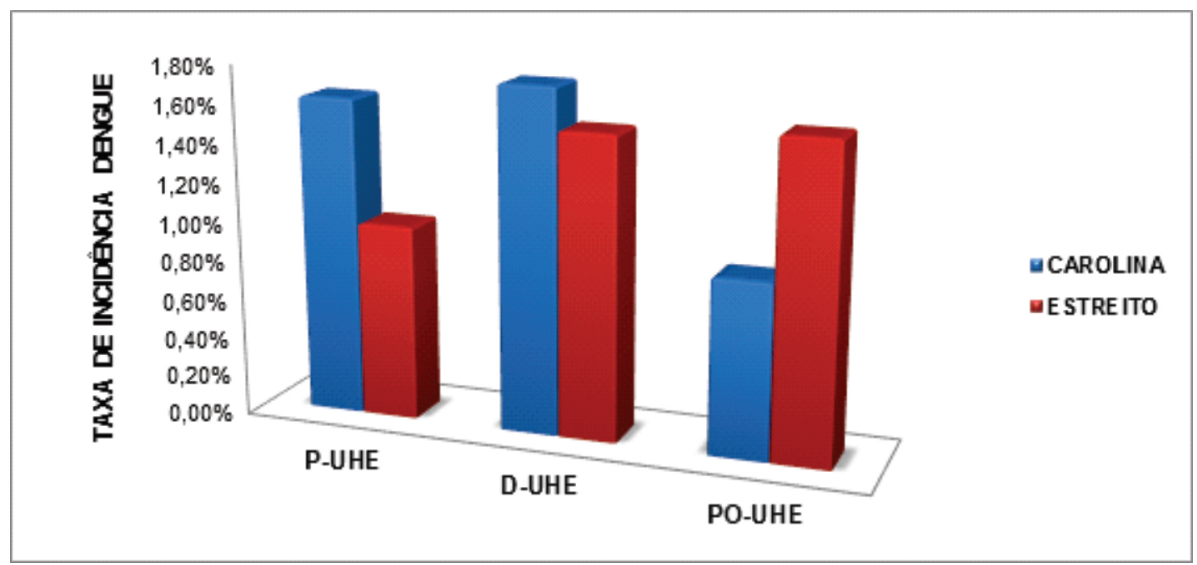

Legenda: P-UHE - Pré UHE; D-UHE - Durante UHE; PO-UHE - Pós UHE. Fonte SINAN, 2013.

Apesar de tais fatos, de acordo com análise ANOVA, a Dengue não apresentou variações significativas em relação aos períodos e às duas cidades estudadas (Figura 4).

Figura 4 - Variação da ocorrência de Dengue em Estreito e Carolina (MA) antes, durante e após a implantação da UHE Estreito.

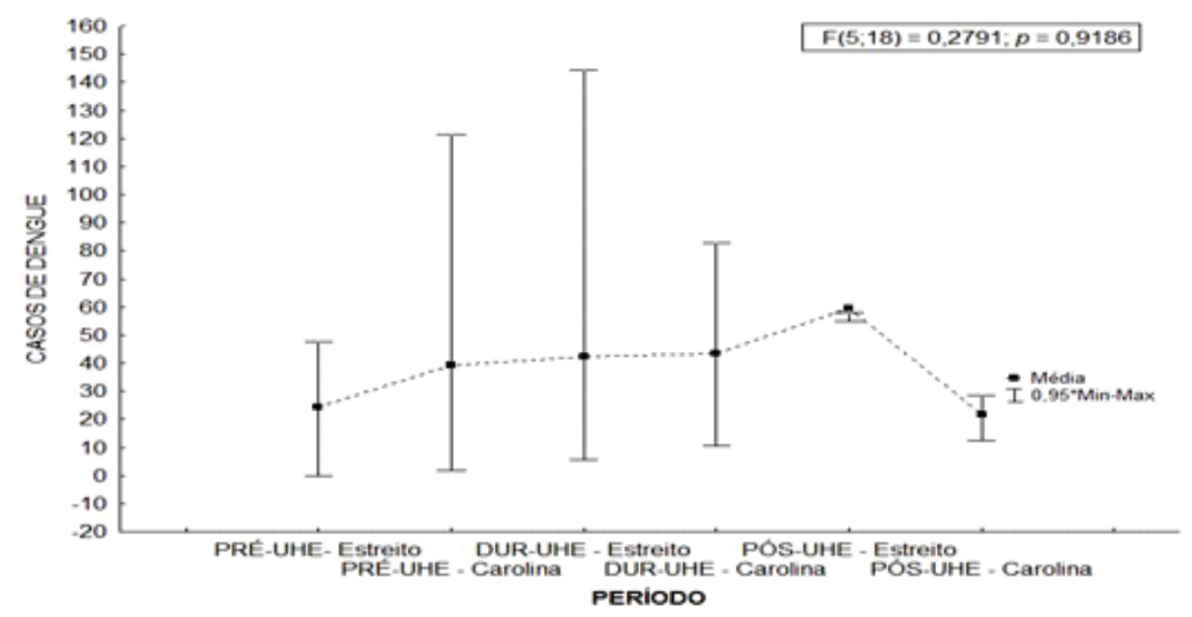

\section{Leishmaniose Tegumentar Americana}

Em diversos estudos foi possível analisar que não somente após a formação do lago reservatório, mas também durante o processo de desmatamento às 
margens da área a ser alagada, grande quantidade de doenças, foram responsáveis por casos de epidemia em regiões impactadas pela construção de UHEs principalmente as transmitidas por vetores e as de veiculação hídrica (FERRETE et al., 2004; CRUZ, 2008), como a incidência de Leishmaniose Tegumentar Americana (LTA) na área de estudo (Figura 5).

O caso da UHE Tucuruí (PA) demonstra a grande importância de um acompanhamento epidemiológico tanto preventivo quanto de longo prazo após o término da obra, onde a LTA se fez presente em todos os municípios afetados. O fato preocupante é que, mesmo vários anos após a conclusão da obra, o número de casos foi aumentando significativamente, evoluindo de 18 casos no ano de 1983, a praticamente 1.000 em 1995, dez anos após a conclusão da UHE (MOURA, 1998).

Figura 5 - Taxa de incidência Leishmaniose Tegumentar Americana em Estreito e Carolina (MA) - antes, durante e após a UHE.

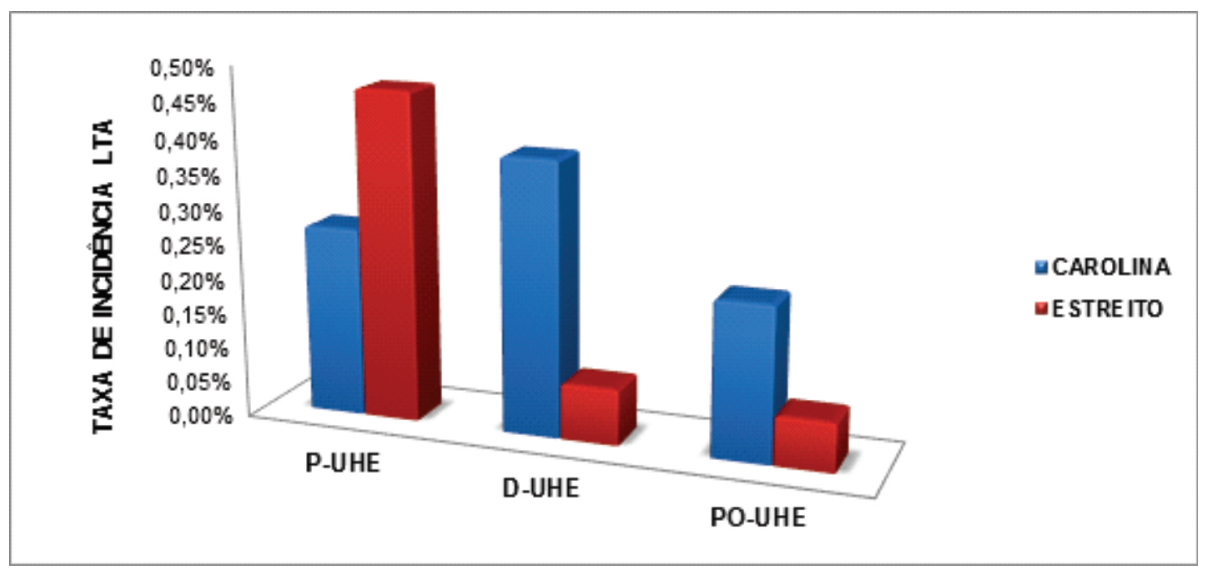

Fonte: SINAN, 2013.

A LTA, por sua vez, em relação às variações da ocorrência de casos, tanto as duas cidades como os períodos analisados apresentaram variações significativas entre si, conforme análise de ANOVA, em que o valor de $\mathrm{p}<0,05$ (Figura 6). 
Figura 6 - Variação da ocorrência de LTA em Estreito e Carolina (MA) - antes, durante e após a UHE.

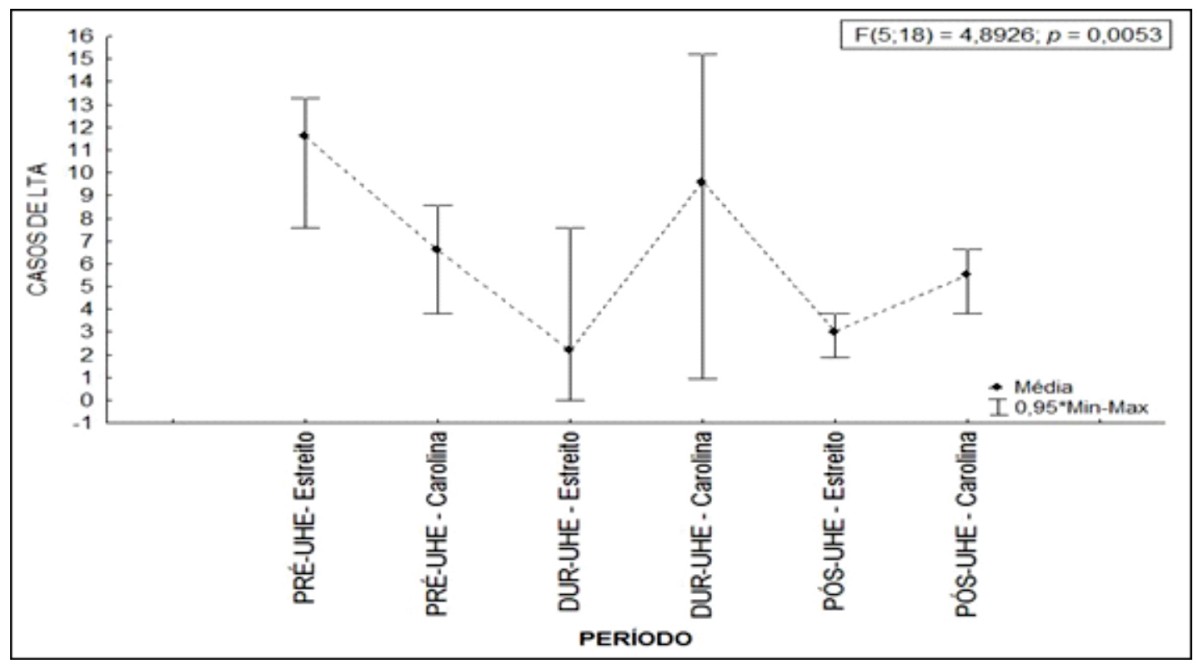

\section{Leishmaniose Visceral}

Tanto Carolina como Estreito apresentaram condições ideais para a manutenção do ciclo epidemiológico da Leishmaniose Visceral devido ao crescimento populacional desordenado, propiciando o desenvolvimento de grandes áreas periféricas, sem que os serviços públicos essenciais conseguissem abranger essas áreas com a mesma velocidade com que elas cresciam.

O município de Carolina, por sua vez, apresentou crescimento na incidência de casos, principalmente no período durante a construção da UHE. Dentre os principais fatores que podem ter influenciado, destacam-se a grande massa trabalhadora em áreas rurais nos desmatamentos às margens do rio e os remanejamentos populacionais de áreas ribeirinhas, sejam elas no perímetro urbano ou no rural, adicionados ao crescimento de áreas periféricas da cidade, tanto por trabalhadores da UHE quanto por moradores remanejados de áreas ribeirinhas (Figura 7).

Estreito, no entanto, apresentou leve oscilação em relação ao período, com maior incidência de casos, tendo o período após o enchimento total do lago reservatório como o de maior índice da doença (Figura 7). 
Figura 7 - Taxa de incidência Leishmaniose Visceral.

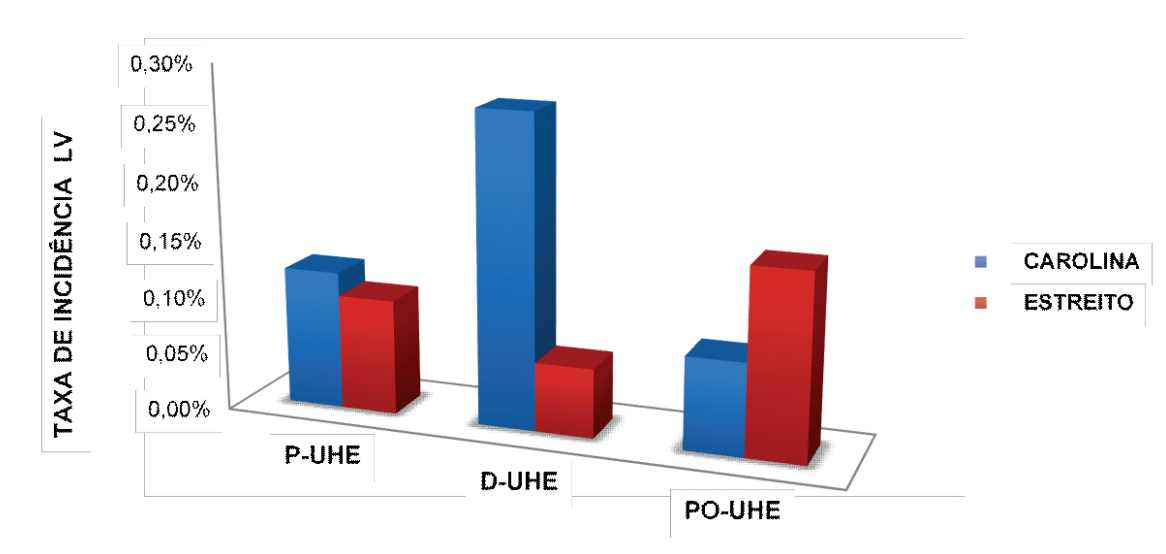

Fonte: SINAN, 2013.

Assim como a Dengue, a LV não apresentou bruscas variações de casos em relação às duas cidades e aos períodos analisados, onde o valor de $\mathrm{p}>0,05$ (Figura 8).

Figura 8 - Variação da ocorrência de LV em Estreito e Carolina (MA) - antes, durante e após a UHE.

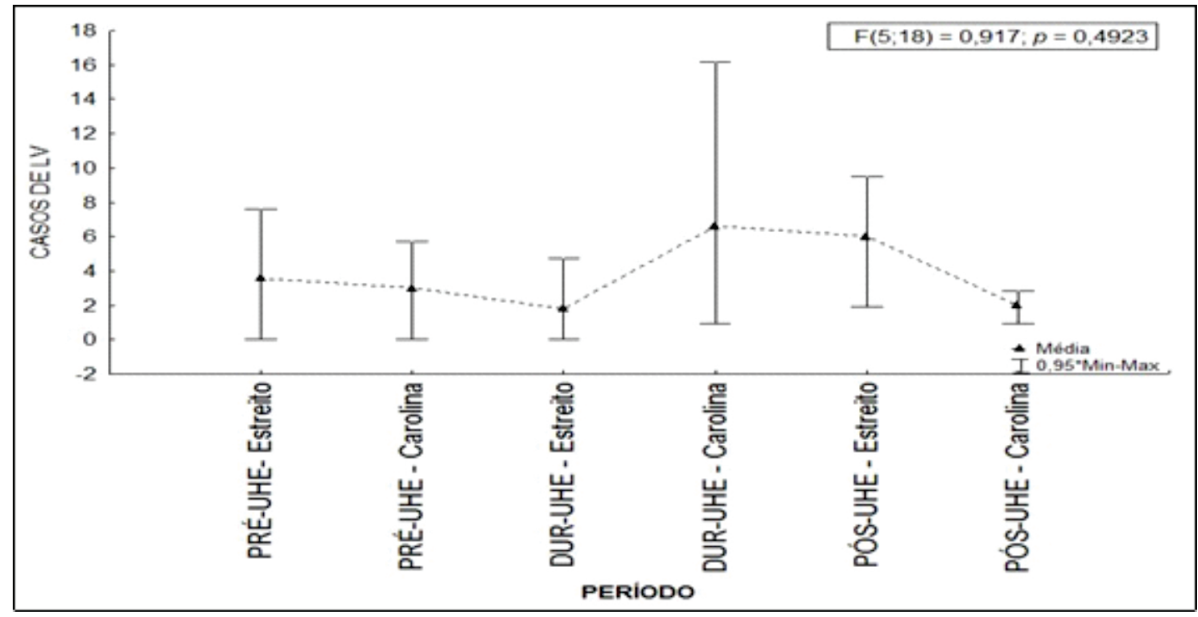

Outras doenças como a Doença de Chagas, a Febre Amarela e a Malária, apesar de não apresentarem casos notificados nos anos de estudo, não devem ser ignoradas por estudos epidemiológicos e programas de saúde pública, não só 
pela identificação de vetores destas morbidades na região, mas pelo fato de tais doenças terem sido responsáveis por epidemias em diversas outras regiões abaladas pela construção de UHEs, além de apresentar todos os indivíduos necessários para conclusão do ciclo epidemiológico das doenças mencionadas: Vetores hospedeiros intermediários - hospedeiros definitivos (KATSURAGAWA et al., 2008) (Tabela 2).

Tabela 2 - Espécies de interesse médico coletados nas campanhas do Programa de Controle de Vetores da UHE Estreito, e agravos potencialmente vetorizados.

\begin{tabular}{|c|c|}
\hline TAXA & AGRAVO \\
\hline \multicolumn{2}{|l|}{ Filo Arthropoda } \\
\hline \multicolumn{2}{|l|}{ Classe Insecta } \\
\hline \multicolumn{2}{|l|}{ Ordem Diptera } \\
\hline \multicolumn{2}{|l|}{ Família Culicidae } \\
\hline \multicolumn{2}{|l|}{ Subfamília Anophelinae } \\
\hline Anopheles (Nyssorbynchus) darlingi & Transmissor primário de Malária \\
\hline Anopheles (Nyssorbynchus) evansae & Transmissor secundário de Malária \\
\hline Anopheles (Nyssorbynchus) nuneztovari & Transmissor secundário de Malária \\
\hline Anopheles (Nyssorbynchus) triannulatus & Transmissor secundário de Malária \\
\hline \multicolumn{2}{|l|}{ Subfamília Culicinae } \\
\hline Culexsp. & Filariose \\
\hline Psorophora ferox & Encefalites virais \\
\hline Psorophora sp. & Algumas arboviroses \\
\hline Sabethes (Sabethoides) chloropterus & $\begin{array}{l}\text { Transmissor de Febre Amarela } \\
\text { Silvestre }\end{array}$ \\
\hline \multicolumn{2}{|l|}{ Família Psychodidae } \\
\hline \multicolumn{2}{|l|}{ Subfamília Phlebotominae } \\
\hline Lutromyia (Nyssomyia) antunesi & Leishmaniose Tegumentar \\
\hline Lutzomyia (Nyssomyia) flaviscutellata & Leishmaniose Tegumentar \\
\hline Lutromyia (Lutromyia) longipalpis & Leishmaniose Visceral \\
\hline Lutromyia (Psychodopygus) davisi & Leishmaniose Tegumentar \\
\hline Lutromyia (Psychodopygus) welcomei & Leishmaniose Tegumentar \\
\hline Lutromyia sp.1 & Gênero transmissor de leishmanioses \\
\hline Lutzomyia sp.2 & Gênero transmissor de leishmanioses \\
\hline Triatoma psendomaculata & Doença de Chagas \\
\hline
\end{tabular}


Não pode ser descartada a hipótese da ocorrência de casos dessas doenças na região, sendo notificados em outras cidades, devido à pouca estrutura em serviços de saúde, fazendo com que os cidadãos, à procura de atendimento de maior qualidade, dirijam-se aos grandes centros urbanos, já orientados a informar que são residentes no município de atendimento, para a otimização da assistência, o que gera a subnotificação para o município de origem e uma notificação indevida para a área de atendimento (GOMES, H., 2014).

\section{CONCLUSÃO}

As incidências das morbidades estudadas variaram independentemente entre as cidades estudadas, não havendo semelhança entre os períodos com maior índice de casos nos períodos estudados, em ambas as cidades.

A Dengue e Leishmaniose Visceral não apresentaram variações consideráveis entre as duas cidades e nos períodos estudados, não ocorrendo o mesmo com a Leishmaniose Tegumentar Americana, pois esta doença apresentou variações consideráveis entre as cidades e os períodos estudados.

As análises dos dados por meio do cálculo de incidência e de variância (ANOVA) indicam que, até o momento, os dados obtidos neste estudo não sugerem a interferência direta e exclusiva entre a construção da UHE e as alterações epidemiológicas consideráveis das morbidades estudadas, haja vista que após a construção da hidrelétrica não houve variações significativas nos índices das doenças.

Torna-se indiscutível a importância da realização de estudos epidemiológicos adicionais na área de influência direta da UHE, em relação à ocorrência de outras morbidades e seus fatores associados, pelo fato de que pouco tempo se passou desde o enchimento total do lago reservatório, e considerando que os dados obtidos neste estudo não são suficientes para relacionar a construção do empreendimento com os índices de zoonoses. 


\section{REFERÊNCIAS}

AMARAL, A. M. R.; MACHADO, C. J. S.; MOULTON, T. P. Construção de Hidrelétricas e Saúde Pública no Brasil: Síntese e Crítica de um Processo. In: Encontro Nacional da ANPPAS, 5, 2010. Florianópolis. Anais... Florianópolis: ANPAS, 2010. 20p.

ANEEL-Agência Nacional de Energia Elétrica. Atlas de Energia Elétrica no Brasil. Brasília, DF: ANEEL, 2002. 153p.

BRASIL. Ministério da Saúde. Diagnóstico rápido nos municípios para vigilância entomológica do Aedes aegypti no Brasil: metodologia para avaliação dos índices de Breteau e Predial. Brasília: MS, 2005. 60 p.

CESTE. UHE Estreito Dados Técnicos. 2013. Disponível em: <http://www.uheestreito.com.br/ver_secao.php?session_id=73 > Acesso em: 20 abr. 2013.

COUTO, R. C. S. Hidrelétricas e saúde na Amazônia: um estudo sobre a tendência da malária na área do lago da hidrelétrica Tucuruí, PA. 1996. 135f. Dissertação (Mestrado em Saúde Pública) - Escola Nacional de Saúde Pública, Rio de Janeiro, 1996.

CRUZ, M. F. R. Estudo epidemiológico de Leishmaniose Tegumentar Americana (LTA) no município de Itambaracá, região norte do estado do Paraná, Brasil, em áreas de influência do complexo hidrelétrico na bacia do rio Paranapanema, 2004-2006. 2008. 168f. Tese (Doutorado em Epidemiologia) - Faculdade de Saúde Pública, Universidade de São Paulo, São Paulo, 2008.

DACHERY, J. M.; SEVERGNINI, K.; BARBISAN, A. O. Energia elétrica: principal fonte energética do País e a UHE Foz do Chapecó. Unoesc \& Ciência, Joaçaba, v. 1, n. 1, p. 31-38, 2010.

FERRETE, J. A.; LEMOS, J. C.; LIMA, S. C. Lagos artificiais e os fatores condicionantes e determinantes no processo saúde-doença. Caminhos de Geografia, v. 5, n. 13, p. 187 200, 2004.

GOMES, H. Perfil epidemiológico de zoonoses nos municípios afetados diretamente pela usina hidrelétrica Estreito (MA). 2014. 76f. Dissertação (Mestrado em Ciências Ambientais e Saúde) - Pontifícia Universidade Católica de Goiás, Goiânia, 2014.

IBGE. Instituto Brasileiro de Geografia Estatística. Maranhão. 2013. Disponível em: http:/ / cidades.ibge.gov.br/xtras/uf.php?lang=\&coduf $=21 \&$ search=maranhao. Acesso em: 10 maio 2013.

KATSURAGAWA, T. H.; GIL, L. H. S.; TADA, M. S.; LUIZ, S. H. P. Endemias e epidemias na Amazônia: malária e doenças emergentes em áreas ribeirinhas do Rio Madeira. Um caso de escola. Estudos Avançados, v. 22, n. 64, p. 111-141, 2008.

LANA, M.; TAFURI, W. L. Doença de Chagas: Parasitologia Humana. 11. ed. São Paulo: Atheneu, 2005. 494 p. 
MOURA, R. C. S. Programa de educação em saúde e vigilância epidemiológica associado à expansão da obra de complementação da UHE Tucuruí. Documento Interno. Brasília, DF: Eletronorte, 1998.

NATURAE. Programa de Controle de Vetores. Relatório Técnico Interpretativo da Usina Hidrelétrica de Estreito (MA). Goiânia: [s.n.], 2011.

OLIVEIRA, F. A. S.; HEUKELBACH, J.; MOURA, R. C. S.; ARIZA, L. J. A. N. R.; GOMIDE, M. Grandes Represas e seus impactos em Saúde Pública. Efeitos a Montante. Cadernos de Saúde Coletiva, Rio de Janeiro, v. 14, n. 4, p. 1-685, 2006.

PEDUZZI, P. Apreensão de crack aumenta $\mathbf{9 0 0 \%}$ em município próximo a Belo Monte. Agência Brasil Notícias, 2013. Disponível em: <http://agenciabrasil.ebc.com. br/noticia/2013-02-15/apreensao-de-crack-aumenta-900-em-municipio-proximo-belomonte >. Acesso em: 20 nov. 2013.

SILVA JR. N. J.; SILVA, H. L. R.; RODRIGUES, M. T. U.; VALLE, N. C.; COSTA, M. C.; LINDER, E. T.; JOHANSSON, C.; SITES JR., J. W. A fauna de vertebrados do vale do alto rio Tocantins em áreas de usinas hidrelétricas. Estudos - Vida e Saúde, v. 32, n. 1, p. 57-102, 2005.

SINAN. Sistema de Informação de Agravos de Notificação. Secretaria de Vigilância em Saúde, 2013. Disponível em: <http://dtr2004.saude.gov.br/sinanweb/. Acesso em: 26 ago. 2013.

ZAR, J. H. Bioestatistical Analysis. 4. ed. New Jersey: Prentice-Hall; Upper Saddle River, 1999. 
